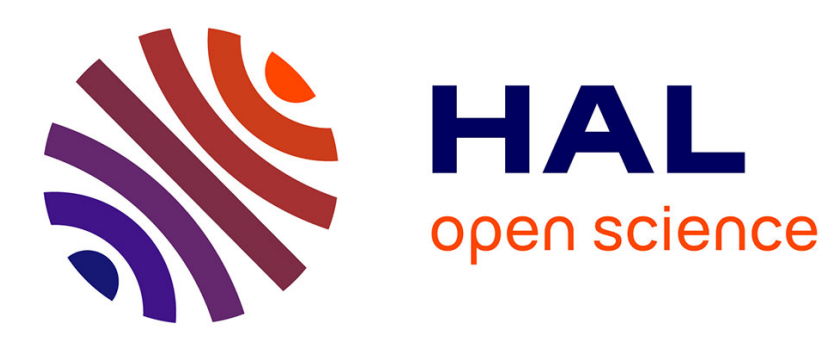

\title{
L'école, lieu de passage et de transmission : un regard anthropologique et clinique
}

\author{
Françoise Hatchuel
}

\section{To cite this version:}

Françoise Hatchuel. L'école, lieu de passage et de transmission : un regard anthropologique et clinique. Nouvelle revue de psychosociologie, 2010, 9 (1), 10.3917/nrp.009.0105 . hal-01658585

\section{HAL Id: hal-01658585 \\ https://hal.parisnanterre.fr/hal-01658585}

Submitted on 7 Dec 2017

HAL is a multi-disciplinary open access archive for the deposit and dissemination of scientific research documents, whether they are published or not. The documents may come from teaching and research institutions in France or abroad, or from public or private research centers.
L'archive ouverte pluridisciplinaire HAL, est destinée au dépôt et à la diffusion de documents scientifiques de niveau recherche, publiés ou non, émanant des établissements d'enseignement et de recherche français ou étrangers, des laboratoires publics ou privés. 


\title{
L'école, lieu de passage et de transmission: un regard anthropologique et clinique
}

\author{
Françoise Hatchuel
}

Dans le contexte d'insécurité et de changement qui prévaut dans nos sociétés, la liste des missions supposées de l'école ne cesse de s'allonger : enseigner correctement les savoirs fondamentaux, fournir une formation professionnelle adéquate, transmettre une culture, former des citoyens et des citoyennes, éduquer au goût, au respect, à l'environnement, au vivre-ensemble... Catalogue de plus en plus hétéroclite, où chacun(e) semble projeter ses désirs et ses angoisses pour l'avenir, tandis que la pression exercée sur les résultats scolaires ne cesse d'augmenter, les enfants d'aujourd'hui se retrouvant priés de triompher, si possible brillamment, des différentes épreuves (dans tous les sens du terme) auxquelles les soumet l'école, sauf à se retrouver étiquetés " en échec scolaire " à 4 ans et demi ${ }^{1}$. En cohérence avec cette inflation des attentes, les critiques fusent également : programmes trop chargés et

1. Le propos est authentique, et qui plus est adressé par une enseignante "expérimentée » à une jeune stagiaire à propos d'un de ses élèves. Nos étudiant(e)s les plus jeunes se laissent d'ailleurs facilement enrôler dans ce système ultracompétitif alors que, notamment dans le cadre de notre master de formation à l'intervention et à l'analyse des pratiques, où nous les invitons à revisiter leur biographie dans une perspective d'élaboration clinique, les plus ancien(ne)s témoignent d'une école où, dans les années 1960 ou 1970, il leur était avant tout demandé de « tenir sa place » à peu près honorablement. 
inadaptés, enseignant(e)s qui ne tiendraient compte ni de la demande sociale, ni de celle de leurs élèves, sécurité non assurée, etc.

Peut-être, alors, faut-il se réinterroger sur le rôle et les fonctions de l'école, non pas en partant du système scolaire que nous connaissons, tel qu'il a été mis en place en Occident au fil des siècles précédents, mais en se plaçant dans une perspective anthropologique plus large afin de questionner les enjeux de la transmission qui se posent à toute société. À quel besoin l'école répond-elle ? Qu'est-ce qui conduit, pour ce faire, à adopter le même dispositif pour toutes les tranches d'âge en rassemblant plusieurs heures par jour l'ensemble des jeunes de 3 à 18 ans dans des établissements idoines sous la houlette d'adultes qui se consacrent à plein temps à leur éducation ? En posant ainsi la question, je souhaite insister sur le fait que l'école telle que nous la connaissons constitue une modalité possible de prise en charge des plus jeunes mais qu'elle n'est pas la seule, n'a pas toujours prédominé et ne prédomine pas forcément dans certaines sociétés, même si le modèle tend à s'étendre. Je pense bien sûr à Ivan Illich (1971) et à sa dénonciation de la lourdeur et la normativité du système adopté par les sociétés occidentales, tout en

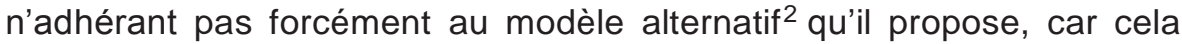
reviendrait, me semble-t-il, à clore le débat avant même de l'avoir réellement ouvert.

Tout l'enjeu me semble en effet se situer là : pouvons-nous supporter, alors même qu'il s'agit de nos enfants, donc de notre survie symbolique, de ne pas avoir de certitude ? Pouvons-nous accepter que nos modes de faire éducatifs s'inventent, aussi, dans la rencontre et le tâtonnement, ce qui ne signifie pas pour autant le chaos et l'angoisse ? Entre les fausses solutions toutes faites et l'anomie sauvage, quelle place pouvons-nous trouver pour un espace d'élaboration collective instituant ${ }^{3}$ ? Je voudrais donc proposer ici un changement de point de vue, en partant non pas de l'école telle qu'elle est et en cherchant à l'améliorer, mais en revenant aux besoins fondamentaux de l'espèce humaine confrontée à la lourde tâche d'amener ses enfants à trouver une place dans le monde. Cette tâche est d'ordinaire évoquée sous le terme de "socialisation » mais je proposerai plutôt celui d' « anthropologisation ", en m'appuyant pour ce faire à la fois sur certains travaux anthropologiques et sur la théorisation de la psychanalyste Piera Aulagnier.

\section{« L'ANTHROPOLOGISATION » : PISTES POUR UNE DÉFINITION}

C'est à partir de sa clinique de sujets psychotiques que cette auteure a élaboré une théorie du psychisme humain se développant en trois

2. Sous forme de mise en réseau et d'échanges libres de savoirs, dont on peut retrouver l'esprit dans les réseaux d'échanges de savoirs.

3. Au sens de Castoriadis (1975). 
temps : l'originaire, le primaire et le secondaire. À la conception « classique » qui place les processus primaires sous le signe du fantasme et associe les processus secondaires à la symbolisation, elle ajoute la notion d'« originaire ", qui lui est propre, et qu'elle conçoit comme une formation très archaïque du psychisme, résultant de ce qui lui est parvenu trop tôt pour pouvoir être mis en image ou en mots. Elle utilise alors le terme de «pictogramme » pour désigner ces éléments «bruts » qui restent comme enkystés dans le psychisme (Aulagnier, 1975). L'archétype du pictogramme se situe dans la rencontre «bouche-sein » et la façon dont elle est reçue par le nouveau-né, si bien que les processus originaires seront déterminants pour soutenir l'investissement du sujet, c'est-à-dire sa capacité à maintenir un désir vivant (voir Aulagnier, 1982/1986). La façon dont j'interprète ces travaux me conduit alors à penser que pour que le Je, au sens où elle l'entend, puisse advenir, le sujet doit pouvoir soutenir une croyance minimale, et qui lui est transmise, en une certaine capacité de vivre malgré et avec la conscience de la mort.

Je pense en effet que cette conscience est une des caractéristiques de l'espèce humaine et que, pour y faire face, les sociétés ont développé des étayages symboliques venant soutenir les psychismes des sujets. Ce sont ces étayages, me semble-t-il, qui permettent au sujet de grandir comme humain et aux adultes de l'y aider. Si nous regardons par exemple les différents rites de naissance à l'œuvre dans de nombreuses sociétés (voir par exemple Bonnet/Pourchez, 2007), nous constatons qu'il s'agit toujours à la fois de prévenir les dangers qui guettent le nouveau-né (et donc de contenir l'angoisse des adultes quant à sa fragilité) et de l'instituer comme sujet appartenant à la communauté des humains, communauté qui le reçoit comme tel et atteste de cette appartenance.

Dans une société traditionnelle, cette réception par la communauté est pensée en même temps que la place sociale qu'occupera l'enfant, en fonction notamment du lignage de ses parents et de ce que Bourdieu appelle leur " capital social ». Notamment mais pas entièrement, car une société, aussi codifiée soit-elle, autorise toujours un certain « jeu » dans les places que les sujets pourront occuper. Les rituels de passage à l'âge adulte constitueront un des temps où ce jeu pourra se percevoir. Nous y reviendrons. Mais dans nos sociétés hypermodernes, les places sont de plus en plus incertaines : non pas que le capital social ne soit plus efficient, mais plutôt que la société devenant elle-même incertaine, avoir une relative garantie d'être en position haute ne dit rien de ce à quoi ressemblera sa vie, sans parler de ceux et celles dont la position de départ préfigure plutôt une position basse. Les sujets ne peuvent donc plus se projeter dans un avenir à peu près imaginable pour se rassurer sur la possibilité pour eux de vivre. II me semble qu'il y a là un changement fondamental : là où la société traditionnelle pense dans le même mouvement à la fois la possibilité de vivre et la forme de cette possibilité, je crois que nos sociétés exigent aujourd'hui de délier les deux. Comme 
si ce que Piera Aulagnier appelle la " capacité à investir ", c'est-à-dire à maintenir un Je désirant et vivant, pouvait de moins en moins s'appuyer sur les éléments imaginaires et symboliques et ne pouvait plus compter que sur les processus originaires et les formes (ou plutôt les " nonformes ") pictogrammiques reçues. C'est à partir de la façon dont il a été reçu, bercé, nourri, accueilli par les adultes mais aussi par le reste du monde extérieur (chaud, froid, bruyant, rapide, lent, fluide, heurté, etc.) que le sujet développera sa croyance à pouvoir se constituer en sujet humain susceptible de vivre au sein d'une communauté. C'est ce processus que je propose d'appeler « anthropologisation », placé sous le signe du pictogramme, alors que la socialisation serait de l'ordre du secondaire et du symbolique, et l'adhésion à l'imaginaire collectif ${ }^{4}$ de l'ordre du primaire ${ }^{5}$.

Nous pouvons comprendre, me semble-t-il, quelque chose de ce que serait l'anthropologisation à travers le beau travail d'observation d'un foyer de jeunes filles mené par l'anthropologue Eric Chauvier (2008), lorsqu'il évoque Joy, une adolescente qui l'a particulièrement touché : " Joy est obstinément en dehors de la conversation. Je veux parler de l'intonation de sa voix et de son inefficacité fondamentale à suggérer la colère » (p. 37) et, pourrions-nous dire, la plupart des sentiments qu'elle cherche pourtant à inspirer. Joy " n'est pas là ", et elle-même « ne semble pas croire en "l'impression de réalité » que ses propos sont censés traduire et que tout locuteur se forme avant de prendre la parole » (ibid., p. 49-50). Si bien que "la situation de Joy évoque les films d'épouvante " (ibid., p. 85) et que la personne même de Joy lui semble de l'ordre de l'énigme, fantomatique et absente au monde. II me semble alors que ces sensations (voix, rythmes, tenue corporelle) par lesquelles passe ou non cet " effet de présence " ont exactement à voir avec ce que Piera Aulagnier appelle «le pictogramme » et que c'est bien là que se situe le travail d'anthropologisation.

\section{UNE INTERPRÉTATION ANTHROPOLOGIQUE DE LA FONCTION DE L'ÉCOLE}

Et pour ce faire, les parents ne suffisent pas. "II faut plus qu'un homme et une femme pour faire un enfant ", nous dit Maurice Godelier (2007) à partir d'une étude des mythes concernant la conception. II souligne ainsi que, dans tous ces « grands récits " (pour reprendre les termes de Jean-François Lyotard, 1979), l'union d'un homme et d'une femme donne naissance à un fœtus, qui devra ensuite, selon les mythes, recevoir une âme, être investi par un esprit, accueillir la réincarnation

4. Voir par exemple Giust-Desprairies, 2003a.

5. J'ai tenté, dans un travail à paraître (Hatchuel, 2010) de montrer en quoi une danse comme le tango argentin, danse créée par de très jeunes hommes exilés, pouvait être interprétée comme une façon de recréer, à travers des sensations corporelles très archaïques, un monde accueillant et susceptible d'être investi. 
d'un(e) ancien(ne), etc. Les modalités peuvent différer mais, dans tous les cas, les deux parents donnent naissance à une entité biologique qui devra ensuite être " humanisée ». Je crois alors que la même formule peut être reprise pour le deuxième grand passage de la vie humaine : il faut plus qu'un homme et une femme pour faire un adulte, et aucune société n'est assez folle pour laisser les seuls parents s'acquitter de cette tâche. Toutes instituent, sous des formes diverses et parfois violentes, des processus de séparation et de médiation venant signifier que les forces à venir que constituent les enfants sont avant tout au service de la société qui doit se reproduire, les parents se voyant simplement confier une partie de la tâche éducative. L'ouvrage de Suzanne Lallemand (1993) donne un assez bon aperçu de la façon dont, par exemple, dans de nombreuses sociétés, les enfants vont circuler d'une famille à l'autre. Un cas extrême se rencontre chez les Inuits, où près d'un tiers des enfants, et notamment les aîné(e)s des jeunes couples, grandissent dans un foyer qui n'est pas celui de leurs parents biologiques. Bernard Saladin d'Anglure (1988) compare ce «partage d'enfants » au partage du gibier ou à l'échange rituel de conjoints, interprétant l'ensemble comme des outils au service de la cohésion du groupe :

" On a [peu] écrit sur ces rites de passage accompagnés de violence physique ou morale [...] exclusion des garçons du partage de leurs premiers gibiers ; violence aussi que constitue le prélèvement d'un nouveau-né en début de mariage et l'obligation pour les jeunes époux de participer aux échanges collectifs de conjoints. II s'agissait pour le groupe, pensons-nous, de se prémunir de tout excès d'individualisme chez les jeunes adultes, surtout lorsque la chance les favorisait dans la production de gibier et la procréation [...] Une part sociale de 15 à $30 \%$ des gibiers, des aliments, des enfants et de la sexualité conjugale n'était certainement pas trop cher payé cette assurance pour le futur, contrôlée par les anciens, par ceux qui nomment, qui transfèrent, qui gèrent et qui échangent le capital social et symbolique de la société » (ibid., p. 161).

Le travail resterait à faire pour étudier finement la diversité de ces modalités de séparation mais, comme le montre très joliment Alma Gottlieb (2000), les enfants, surtout les plus jeunes, restent le point aveugle de l'anthropologie. Les travaux ci-dessus me conduisent néanmoins à faire l'hypothèse d'un lien avec les systèmes de parenté : il semblerait que les sociétés qui privilégient une lignée (que celle-ci soit paternelle ou maternelle) tendent à confier l'éducation à la dite lignée et/ ou ses représentants, tandis que les sociétés dont le système de parenté est dit cognatique, c'est-à-dire où « les relations aux ascendants du père et de la mère sont reconnues comme équivalentes " (Godelier, op. cit., p. 156) reconnaîtraient la famille nucléaire comme unité de base, à la condition qu'une médiation vienne rappeler la loi du groupe. On peut alors imaginer que l'école vient, à cet égard prendre la place, dans nos sociétés, de la circulation d'enfants chez les Inuits. 
CIRCULATIOND'ENJEUXPSYCHIQUES:

DÉNI DE LA MORT ET COPOUVOIR ENTRE GÉNÉRATIONS

Signifier à l'enfant dans un premier temps qu'il est reçu dans la communauté des humains puis que, dans une organisation sociale donnée, une place lui adviendra par laquelle il contribuera à l'effort de production de la communauté : du point de vue de l'enfant, toute institution éducative doit être garante de cela, en trouvant notamment des modalités de régulation et d'attribution des différentes places possibles. Du point de vue de la société, le système mis en place doit en garantir la pérennité. Une société qui ne trouverait pas comment faire en sorte que ses enfants contribuent à la reproduire ne serait pas destinée à un très grand avenir...

Or la tâche n'est pas facile, du fait notamment de la grande fragilité des enfants. Faire grandir des enfants c'est, comme je l'ai souligné par ailleurs (Hatchuel, 2006), avant tout soutenir psychiquement pour eux l'idée d'un avenir possible, et je fais l'hypothèse que toute organisation sociale se structure en partie à partir de cette nécessité : l'éducation se soutient d'un ensemble de représentations imaginaires, sous forme à la fois de mythes et de rôles dévolus à chacun(e) pour, d'une part parvenir à son but, d'autre part ne pas être pensée comme pouvant échouer. En effet, si nous admettons l'idée qu'il y va de la survie de la société, et donc fantasmatiquement de celle des adultes éducateurs, alors l'éducation s'avère une défense redoutablement efficace contre l'angoisse de mort : je crois donc que nous soutenons psychiquement l'idée d'une éducation qui ne saurait échouer parce que c'est ce qui nous soutient dans l'idée que nous ne saurions mourir, ou en tout cas pas totalement, et que cet étayage s'appuie sur des éléments très archaïques de type pictogrammique.

L'enfant, porteur de valeurs, d'attentes et de capacités d'agir futures sera donc investi par des adultes qui parviendront, grâce à cet investissement et à ce qui le soutient socialement, à le mener à une place sociale de compromis entre les différents enjeux des sujets en présence et les exigences sociales. Dans une société traditionnelle, le " phénomène-autorité » règle le problème de façon assez efficace, comme l'a magistralement montré Gérard Mendel (1971) : les anciens détiennent le pouvoir, les jeunes s'adaptent. Le «pacte » tient parce que l'expérience et le pouvoir des anciens contribuent effectivement à fournir une place à chacun(e), ce qui n'est plus le cas dans une société de changement. Comme le dit si bien Michaël Singleton (non daté) : "Si les vieux en Afrique ont été respectés au point qu'on ait pu prendre ce respect ritualisé pour une véritable vénération, c'est qu'ils étaient respectables à cause d'un apport spécifique matériellement et moralement indispensable à la survie de leur société » (p. 12). Mais ce sur quoi je voudrais insister, c'est que le «pacte » tient également parce que la réalité biologique et la 
façon dont elle est parlée permettent aux plus jeunes de se projeter dans le moment où le pouvoir leur reviendra. Dans nos sociétés où la mort est à la fois réellement repoussée et fantasmatiquement évacuée, le jeune qui laisserait prise à l'autorité s'y verrait fantasmatiquement soumis jusqu'à la nuit des temps.

Cohabitation de générations : l'effet conjugué du déconditionnement à l'autorité et des progrès de la médecine conduit deux, voire trois générations à devoir se penser comme simultanément au pouvoir. C'est sans doute une révolution anthropologique dont nous n'avons pas fini de mesurer l'ampleur puisqu'il s'agit de s'assumer à la fois dans la filiation (donc dans la continuité) et dans l'égalité (c'est-à-dire la prise en compte des différentes singularités). La philosophe Geneviève Fraysse (1998) montre, à propos des (in)égalités hommes-femmes, à quel point nous confondons égalité (de droit) et similitude, comme s'il fallait, pour avoir les mêmes droits, se penser identiques. Florence Giust-Desprairies (2003b) souligne, de son côté, à quel point cette " égalité " négatrice d'identité constitue un des mythes fondateurs de notre école. Nous pouvons alors appliquer la même grille aux relations entre générations, en la complexifiant du fait de l'enjeu évoqué ci-dessus : transmettre, c'est aussi lutter contre la mort, et donc être tenté de se reproduire à l'identique. C'est ce que j'ai appelé (Hatchuel, 2009a) la «tentation du clone », qui est à la fois celle de la génération qui transmet et celle de la jeune génération qui se voit ainsi rassurée sur la possibilité d'une insertion. Je la conçois comme très liée à la notion d'investissement : c'est parce que nous avons appris à investir un certain mode de faire ${ }^{6}$ comme rendant la vie possible pour nous que nous ne pouvons supporter qu'il disparaisse avec nous. Se situer à la fois dans l'égalité et la transmission, c'est alors accepter que ce qui est soutenant pour nous et nous a permis de faire grandir nos enfants ne sera pourtant pas forcément ce qui les soutiendra eux-mêmes, alors que dans une société traditionnelle, la personne qui transmet peut toujours s'illusionner sur ce qu'il advient de ce qu'elle aura transmis lorsqu'elle ne sera plus là.

\section{« FAIRE COMME SI » : DE L'ÉCART ENTRE PENSÉES ET ACTES}

Car les sociétés traditionnelles sont avant tout des sociétés du « faire comme si ", où les modalités de contrôle centrées sur la scène sociale laissent une place à l'intimité. En effet, si l'essentiel est de " sauver la face » (Goffman, 1974), c'est-à-dire d'adopter un comportement qui permette de tenir une place sur la scène sociale, cela ne dit rien de ce qui se vit émotionnellement pour le sujet. Marthe Coppel (1991) souligne ainsi en quoi la politesse permet à l'enfant de vivre ses « mauvaises

6. J'insiste sur ce terme de «mode » qui regroupe pour moi les trois dimensions : pictogrammique, imaginaire et symbolique. 
pensées » à l'abri d'un comportement codifié qui certifie qu'elles n'envahiront pas la scène sociale. De façon plus profonde peut-être, Christian Geffray (2001) montre comment c'est la structure sociale même des Yanomami qui repose sur l'existence de waïtéri, ces « valeureux guerriers » qui sont censés ne pas craindre la mort, alors même que « nul n'était dupe alors en ceci que personne ne prenait les waïtéri pour les impossibles et divins sujets-sans-peur-qui-ne-sauraient-mentir [...] $\mathrm{Ce}$ dont on ne doutait pas en revanche, c'étaient qu'ils fussent prêts à payer tout le prix, mourir, pour donner à croire qu'ils n'avaient pas peur de mourir » (ibid., p. 44). Parce qu'ils ne craignent pas la mort, ou en tout cas se comportent comme si, si bien que leurs enjeux personnels n'entravent pas leurs actions, la parole des waitéri est crédible et on peut leur confier le rôle du régulateur sur lequel repose la société yanomami. Leur crédibilité porte non pas sur leurs pensées intimes, qui n'appartiennent qu'à eux, mais sur leurs actes, dont nul n'imaginerait qu'ils devraient en quoi que ce soit être en cohérence avec leurs pensées.

Dans un registre un peu différent mais qui me semble significatif de processus similaires, Bernard Saladin d'Anglure (1992) nous montre une société inuite aux rôles sociaux (et notamment sexués) extrêmement codifiés mais auxquels les individus ne sont pas forcément astreints. L'auteur estime ainsi que 20 à $40 \%$ des enfants sont travestis (c'està-dire élevés dans une identité de genre différente de celle de leur sexe biologique) durant leur enfance. Le travestissement peut être total ou partiel, l'enfant portant, par exemple, des pièces de vêtements ou usant d'outils caractéristiques des deux sexes. Son objectif est avant tout d'assurer à la cellule familiale la présence d'adolescent(e)s susceptibles de seconder les parents dans leurs activités productives (notamment chasse et couture). Dans une fratrie de filles, celle qui montrera le plus d'habileté motrice et/ou le plus de désir d'endosser ce rôle sera ainsi élevée en garçon jusqu'à ce qu'un petit frère puisse la remplacer. Dans tous les cas, les jeunes réintègrent à l'âge adulte l'identité de genre correspondant à leur sexe biologique, conservant les habiletés acquises et apprenant, lorsqu'ils et elles se marient, celles correspondant à leur sexe. Ils deviennent donc très polyvalents et sont, à ce titre, très appréciés de la communauté. L'auteur fait, enfin, l'hypothèse (étayée par l'étude d'objets et de récits) que leur faculté à « passer » d'un genre à l'autre les prédispose à un autre passage, celui entre le monde des vivants et celui des esprits, et que c'est parmi eux que sont recrutés les chamans.

Ces différents exemples me poussent à faire l'hypothèse selon laquelle, plus les codes sont solides, plus cela laisse dans une certaine mesure d'espace au sujet. Dans une certaine mesure, car cet espace peut s'avérer très opaque, très silencieux et très contraint. Mais il me semble tout de même que nous pouvons parler d'une solitude quasi anthropologique de nos adolescents et adolescentes qui ne disposent plus des repères traditionnels (héritages, codes, récits, rituels) qui pouvaient les 
guider sur le chemin d'une confrontation à leurs peurs et à l'abri desquels ils pouvaient s'expérimenter relativement librement. La tyrannie de la transparence que subit l'individu contemporain ricoche dangereusement sur nos adolescent(e)s en ne laissant aucune place au tâtonnement.

\section{RITUELS, MYTHES ET FICTION}

C'est une recherche sur " le rapport au savoir de jeunes en difficulté » (Hatchuel, Carlin, Hans, Clerc, 2005) qui nous a fait saisir collectivement quelque chose de l'effet de l'intrusion psychique, qui me semble être caractéristique de nos mondes contemporains, sur la construction de soi des jeunes. Pour ma part, j'ai plus particulièrement approfondi la forme d'intrusion que j'ai intitulée «mise au défi » et que je peux définir comme l'imposition à autrui des épreuves qu'il doit réussir pour se juger digne de valeur, doublée d'une très forte mise en doute de sa capacité à y parvenir. La mise au défi impose donc de l'extérieur le jugement sur luimême du sujet, ce qui n'est pas très nouveau, mais le rôle qu'a toujours tenu le monde extérieur dans la constitution du psychisme est ici comme capté, dans l'interaction, par un seul sujet, et sans doute la nouveauté est-elle là. La mise au défi soupçonne d'emblée le sujet de ne pas pouvoir réussir les épreuves qui l'institueront comme sujet digne de vivre et le somme de prouver, à chaque instant et aux yeux de l'autre soupçonneux, l'inverse. Contrairement aux rituels traditionnels qui instituent qu'à un moment donné, le sujet, parce que telle est la réalité sociale de sa situation, endossera un nouveau rôle qui lui donnera une nouvelle identité, nos jeunes doivent, à tout moment, se prouver eux-mêmes, en l'absence de ces «points de certitude symboliques » qu'évoque Piera Aulagnier (op. cit.). Les points de certitude n'étant plus déposés dans le monde social, c'est au sujet de les réinstituer à chaque instant.

Cette théorisation autour de la mise au défi résulte d'un assez long processus d'élaboration personnelle où la rencontre avec le jeune Stéphane, interrogé dans le cadre de la recherche mentionnée ci-dessus, me renvoyait aux épreuves, initiatiques s'il en est, que j'avais pu vivre lors de concours d'entrée aux grandes écoles (Hatchuel, 2009b). S'est ensuivie toute une réflexion sur la notion de rituels, qui me permet aujourd'hui de distinguer les rituels que je qualifierais de «traditionnels » des « micro-rituels". Les premiers, communément appelés « rites de passage " à la suite des travaux fondateurs d'Arnold Van Gennep (1909), marquent les grandes étapes de la vie : naissance (où le passage est à la fois celui de l'enfant inaugurant sa vie parmi les humains et celui des jeunes parents, surtout s'il s'agit d'un premier-né), entrée dans l'âge adulte, mariage, mort. Victor Turner (1969/1990) a montré qu'ils étaient garants de la cohésion sociale dont ils étaient à la fois le produit et la source. Pourtant, les «nouveaux rituels » tel que Erving Goffman (op. cit.) a pu les repérer marquent aussi des passages : début ou fin d'une 
conversation, marquage d'entrée ou de reconnaissance d'une communauté (comme le montre le beau travail dirigé par Christoph Wulf, 2004), etc. Mais ces passages-là offrent une différence fondamentale avec ceux des rituels de passage traditionnels : ils ne sont pas garantis par le corps social, et n'excluent donc pas le retour en arrière.

Les passages marqués par les rituels traditionnels sont en effet irréversibles : nulle possibilité de dire que l'on n'a pas été initié(e) une fois l'initiation reçue, ou même d'imaginer échouer. L'épreuve sera plus ou moins bien réussie et chaque sujet la traversera différemment, mais elle sera franchie quoi qu'il arrive. Le rituel de passage traditionnel est un processus qui transforme en un résultat social visible et stable un cheminement émotionnel complexe et incertain qui, lui, n'appartient qu'au sujet. Là encore, l'intime et le visible sont déliés, ainsi que la façon dont le sujet négocie l'épreuve et son résultat, alors même qu'il s'agit de traiter avec le plus intime de soi. Comme le souligne Miguel Benasayag (2009) : « La crainte de souffrir est terrible. Dans notre société, il n'y a pas de rite initiatique, pas de rite de passage. Dans tous ces rites - je n'en ai pas passés moi-même, mais j'y ai souvent assisté dans les nations indiennes d'Amérique du Sud - en général le message consiste en cette idée que "tu peux résister à la douleur, au mal, à la tristesse. Tu peux faire avec". À l'inverse de nos sociétés, qui nous font croire qu'il est possible de ne pas ressentir tout ça. " II me semble donc que les rituels traditionnels peuvent être vus comme des « soutiens à l'anthropologisation », marquant, à chaque passage, que le sujet entre de plein droit dans la nouvelle catégorie humaine qui le caractérise.

Rien de tel aujourd'hui où nous devenons nous-mêmes garants de nos propres rituels, devenus presque aussi mouvants que les épreuves qu'ils sont censés étayer ${ }^{7}$, et donc de nos places et de celles d'autrui, dans un processus permanent de négociation. Si Pierre Bourdieu (1982) montre bien que le sujet a toujours négocié avec les rituels, en n'y adhérant jamais totalement, au moins le rituel « fictif », celui que l'on est censé respecter, existe-t-il clairement dans l'imaginaire et permet-il de se situer. Aujourd'hui, ce sont les normes elles-mêmes qui ne cessent d'évoluer. Le jeune couple marié dans une société traditionnelle change de statut (donc de droits et de devoirs), de mode de vie, de position. Les jeunes concubins qui vont déjeuner le dimanche dans la famille de l'un d'entre eux peuvent tout à fait cesser de le faire : il s'agit d'une négociation entre eux et avec les parents, même si les arguments des uns et des autres peuvent quelque peu puiser dans les « usages».

Or, ce que notre recherche sur les jeunes en difficulté nous a montré, c'est que pour ces jeunes, le monde se conjugue de tant d'incertitude qu'ils doivent devenir eux-mêmes leurs propres certitudes. Et ce d'autant plus que notre rapport à la certitude s'est modifié. Lorsque les Yanomami 
" savent » qu'il existe des waïtéri qui n'ont pas peur de la mort, ils ne prétendent pas qu'il s'agit là de la vérité de ces hommes. II s'agit juste d'une vérité, soutenable et soutenue par le corps social et, en tant que telle, opérante. L'existence des waïtéri est un mythe, il est vrai parce que tout se passe comme s'il était vrai, mais il n'est pas réel, et nul Yanomami ne chercherait à le démontrer. Chacun(e) sait la force des histoires qui ne sont néanmoins que des histoires. L'anthropologue africaniste Michaël Singleton (op. cit.) souligne ainsi qu'« il n'y a pas moyen dans ces mêmes langues, et par conséquent dans les mentalités qu'elles articulent, d'opposer "croire" à "savoir" » (p. 6). Il ajoute (ibid.) : « En Tanzanie, "mes" Wakonongo ne disaient pas "nous savons que $2+2=4$ mais nous croyons qu'il y a des sorciers", ils parlaient dans les deux cas de "savoir" (kujua) " si bien que les missionnaires chrétiens ont dû aller chercher un mot arabe pour traduire le credo...

La rationalité scientifique (surtout lorsqu'elle est mal interprétée, car les « vrai(e)s » scientifiques savent bien que les théories ne sont que des modèles aux durées de validité variables) nous pousse à un clivage plus que préjudiciable entre savoirs démontrés et croyances. Non pas qu'il s'agisse de les confondre (quelques siècles de modernité ont amplement prouvé la pertinence de leur distinction) mais plutôt de ne pas ôter toute validité à la croyance, dans le registre qui lui est propre. En effet, si seul le " savoir " (au sens de certitude démontrée) est valable, alors nos jeunes se retrouvent sommés, à l'image du monde, de se démontrer avant de pouvoir se rêver, et de mettre en scène leur intériorité, mise en scène permanente de soi à laquelle l'école participe abondamment. Nous repérons ici la conséquence d'une modernité avide d'emprise, où les savoirs doivent devenir prédictifs et les actes conformes à ce qui en a été prévu, au détriment de la résistance du réel (voir la magistrale réflexion de Gérard Mendel [1998] sur l'acte). La confusion devient totale entre l'acte et l'intentionnalité, attribuant au " hasard » tout effet non désiré consciemment, au mépris de toute réflexion sur les risques pris : une Porsche roulant à 200 à l'heure dans un couloir de bus et fauchant trois personnes ou de l'essence renversée sur une jeune fille qui s'enflamme lorsqu'on approche un briquet sont des « accidents " puisque l'intention consciente n'était pas de donner la $\operatorname{mort}^{8} \ldots$

8. Ces deux exemples, réels, sont tirés de mon expérience d'habitante de Vitrysur-Seine, et l'interprétation en termes d'accident est celle maintes fois entendue dans les débats de jeunes que j'y ai animés. Le deuxième, où la jeune Sohane Benziane est morte brûlée vive alors qu'il s'agissait " seulement » de lui faire peur, a abouti à un verdict qui m'a semblé exemplaire : vingt-cinq ans de réclusion pour « tortures et actes de barbarie ayant entraîné la mort sans intention de la donner ", signifiant que même sans intention, les actes peuvent être gravissimes. 
Je crois en effet que la modernité nous a fait oublier le besoin de croire (voir de Mijolla-Mellor, 2004), c'est-à-dire d'élaborer pour soi puis collectivement (voir Giust-Desprairies, 2003a, op. cit.) un imaginaire susceptible de se remanier mais bénéficiant néanmoins d'une certaine stabilité $^{9}$, au profit d'une conception étroite de la notion de démonstration : il ne s'agit donc pas ici de faire le procès de la démarche scientifique, mais celui de ce qu'un certain Abus de savoir (1977) a pu induire, où les experts deviennent la nouvelle autorité et la rationalité scientifique la valeur. Or, comme le dit si bien Cornélius Castoriadis : "Les fourmis ont une "rationalité" fonctionnelle de loin supérieure à celle de l'homme " (Castoriadis, 1996, p. 111). Notre société vit dans la mystification qu'il y aurait une bonne façon de faire et que nous pourrons la découvrir. Une réflexion sur les différents types de savoirs valorisés selon les sociétés et les modalités d'inclusion qui en découlent (Hatchuel, 2009c) me conduit ainsi à parler de "fiction de soi " que j'opposerai aujourd'hui à la " démonstration de soi ».

Être dans la fiction de soi plutôt que dans la démonstration de soi, ce serait se concevoir de façon perpétuellement évolutive et en même temps ancrée, non pas dans des certitudes a priori mais dans ce que nous comprenons de ce qui, peu à peu, nous constitue et qui n'a bien entendu, rien à voir avec les diktats extérieurs (" et voilà des millions d'individus qui se "personnalisent" en achetant la même lessive ", nous rappelle très justement Cornélius Castoriadis, op. cit., p. 99). Cela a à voir, par contre, avec la façon dont nos parents, puis les autres figures identificatoires, nous ont rêvé et se sont rêvés eux-mêmes, en fonction de ce qui leur est nécessaire pour pouvoir soutenir l'idée de vivre. La dimension pictogrammique me semble ici essentielle : pour pouvoir y vivre, avons-nous appris à rendre le monde rapide, lent, lisse, rugueux, piquant, doux... (les mots ici ne sont que d'imparfaites traductions des sensations emmagasinées) ? Et nous, dans ce monde-là, comment bougeons-nous, comment faisons-nous face aux difficultés, aux frustrations, à l'éloignement, la perte, etc. ? Si les certitudes ne sont plus déposées dans le symbolique, c'est alors peut-être dans les sensations premières que nous pourrons les retrouver. Comme le dit Wittgenstein (1951/2006) : « L'enfant n'apprend pas qu'il y a des livres, qu'il y a des sièges, etc., mais il apprend à aller chercher des livres, à s'asseoir sur un siège, etc. " (ibid., p. 115). Mais ces sensations héritées, en quoi et comment deviennent-elles nôtres ?

Un des enjeux essentiels ici, que souligne bien Piera Aulagnier (1984), c'est l'écart autorisé au discours parental : l'interprétation du monde, en mots et en actes, qui nous est proposée, comment nous

9. Sur la notion de stabilité, et notamment la distinction entre stabilité fixe et stabilité dynamique, on pourra regarder avec bonheur le travail sur Le concept d'enveloppe psychique de Didier Houzel (2005). 
est-elle proposée ? Est-elle elle-même une certitude, une vérité absolue ? "C'est une douillette, un rapide, une contemplative... » Quelle place pour la surprise, le changement, les hésitations, les passages ? Quelle place pour que d'autres adultes puissent participer au rêve, tenir eux aussi un discours sur et avec l'enfant, transmettre quelque chose de leur rapport au monde, dans ses trois dimensions?

\section{CONCLUSION}

C'est exactement dans cet écart-là que vient prendre place l'école, et, parce qu'elle s'appuie sur des outils culturels fortement étayants, elle peut contribuer à le créer. Grandir, ce n'est pas apprendre un métier, mais savoir quel adulte on sera. Et ce savoir-là ne se met pas en fiche, contrairement à ce que nous voudrions croire pour masquer nos propres incertitudes d'adultes. II est le résultat d'un long processus de négociation au sens noble du terme où jeunes et adultes vont pouvoir se rêver et rêver l'autre, et mettre leurs rêves à l'épreuve de la réalité, dans le remaniement et non l'inhibition.

Saurons-nous construire une école (et rien n'est dit de la forme qu'elle pourrait prendre) pour êtres humains, où demeure une place pour l'incertitude, où la distinction se fasse entre ce qui n'appartient qu'au sujet et ce qu'il doit mettre en forme pour pouvoir le partager et s'insérer dans la communauté des humains, où le sujet puisse éprouver la réalité, dans tous les sens du terme, guidé en cela par d'autres sujets acceptant eux-mêmes de vivre leurs épreuves d'humains et d'en témoigner ? Une école où le savoir ne se concevrait plus comme une certitude absolue mais comme un étayage, issu du travail hérité de l'humanité, mais forcément relié à (ce qui ne veut pas dire dépendant de) la subjectivité de chacun(e) ? II y aurait, sans doute, quelque urgence, et avec certitude, quelque intérêt, à le faire... Et si l'analyse, ici, s'est beaucoup appuyée sur l'anthropologie, la psychosociologie reste irremplaçable pour contribuer à trouver comment une telle école pourra se construire.

\section{BIBLIOGRAPHIE}

AULAGNIER, P. 1975 : voir Castoriadis-Aulagnier.

AULAGNIER, P. 1982. "Condamné à investir », Nouvelle revue de psychanalyse, $\mathrm{XXV}$, p. 309-330. Repris dans Un interprète en quête de sens, Paris, Ramsay, 1986, p. 325-358.

BAUMAN, Z. 2006. La vie liquide, Paris, Éditions du Rouergue (édition originale Oxford, 2005).

BEAUMONT, M. 1977. Abus de savoir. Des scientifiques chrétiens s'interrogent, Paris, Desclée de Brouwer.

BENASAYAG, M. 2009. "La liberté, c'est déployer sa propre puissance dans chaque situation ", Interview par K. Margot, disponible sur http ://www. article11.info/spip/spip.php ?article468 
Bonnet, D. ; POURChEZ, L. (sous la direction de). 2007. Du soin au rite dans l'enfance, Toulouse, érès/IRD (préf. Geneviève Delaisi de Parseval).

BOURDIEU, P. 1982. "Les rites comme actes d'institution ", Actes de la recherche en sciences sociales, $\mathrm{n}^{\circ} 43$, p. 58-63, repris dans $C e$ que parler veut dire, L'économie des échanges linguistiques, Paris, Fayard, p. 121-134.

CASTORIADIS, C. 1975. L'institution imaginaire de la société, Paris, Le Seuil.

CASTORIADIS, C. 1996. La montée de l'insignifiance (les carrefours du labyrinthe t. IV), Paris, Le Seuil.

CASTORIADIS-AULAGNIER, P. 1975. La violence de l'interprétation : du pictogramme à l'énoncé, Paris, PUF, coll. « Le fil rouge ».

ChAUVIER, E. 2008. Si l'enfant ne réagit pas, Paris, Allia.

COPPEL, M. 1991. "L'éducateur, le psychanalyste et les mauvaises pensées », Autrement, série «Morales », $\mathrm{n}^{\circ} 2$ : La politesse, vertu des apparences, Paris, Éd. Autrement.

Geffray, C. 2001. Trésors. Anthropologie analytique de la valeur, Paris, Arcanes.

GIUST-DESPRAIRIES, F. 2003a. L'imaginaire collectif, Toulouse, érès.

GIUST-DESPRAIRIES, F. 2003b. La figure de l'Autre dans l'école républicaine, Paris, PUF.

GODELIER, M. 2007. Au fondement des sociétés humaines. Ce que nous apprend l'anthropologie, Paris, Albin Michel.

GofFMAN, E. 1974. Les rites d'interaction, Paris, Minuit.

GOTTLIEB, A. 2000. «Où sont passés tous les bébés ? Vers une anthropologie du nourrisson ", dans J.-L. Jamard, E. Terray, M. Xanthakou (sous la direction de), En substances. Textes pour Françoise Héritier, Paris, Fayard, p. 367-385.

Hatchuel, F. ; CARlin, V. ; CleRC, N. ; HANS, D. 2005. Le rapport au savoir de jeunes en difficulté, rapport final à l'ASEAY (Association pour la sauvegarde de l'enfance et de l'adolescence des Yvelines), dact.

HATCHUEL, F. 2006. « Jeunesse et âge adulte, regard anthropologique », Champs/ Enfances : psychopathologies et clinique sociale, vol. 2, n³-4, automnehiver 2006, p. 15-28.

HATCHUEL, F. 2009a. Du rapport au savoir à la fiction de soi : penser, vivre et faire grandir dans un monde incertain. Anthropologie clinique de la transmission. Note de soutenance pour l'habilitation à diriger les recherches, université Paris-Ouest-Nanterre-La Défense.

HATCHUEL, F. 2009b. "L'impossible intériorité et la mise au défi », Cahiers de psychologie clinique, dossier "Effractions d'adolescence ", $\mathrm{n}^{\circ} 33$ (automne 2009), p. 121-138.

Hatchuel, F. 2009c. Pour une anthropologie modale des savoirs. Essai inédit faisant partie des travaux déposés en vue de l'obtention d'une habilitation à diriger les recherches, université Paris-Ouest-Nanterre-La Défense.

HATCHUEL, F. 2010. " Le tango argentin comme pratique corporelle étayante d'un nouveau mode de lien au monde », L'autre, dossier « Art et soin », automne 2010.

HOUZEL, D. 2005. Le concept d'enveloppe psychique, Paris, In Press.

ILLICH, I. 1971. Une société sans école, Paris, Le Seuil.

LALLEMAND, S. 1993. La circulation des enfants en société traditionnelle : prêt, don, échange, Paris, L'Harmattan.

L'école, lieu de passage et de transmission 
LYOTARD, J.-F. 1979. La condition postmoderne : rapport sur le savoir, Paris, Éditions de Minuit.

MEnDEL, G. 1971. Pour décoloniser l'enfant : sociopsychanalyse de l'autorité, Paris, Payot (6 édition, 1981).

MENDEL, G. 1998. L'acte est une aventure, Paris, La Découverte.

MiJOlla-Mellor, S. (de). 2004. Le besoin de croire. Métapsychologie du fait religieux, Paris, Dunod.

SALADIN D'ANGLURE, B. 1988. "Enfants nomades au pays des Inuit Iglulik ", Anthropologie et sociétés, vol. 12, n², p. 125-166.

SALADIN D'ANGLURE, B. 1992. "Le troisième sexe », La recherche, $\mathrm{n}^{\circ} 245$, p. 836-844.

SINGLETON, M. (non daté). «Du culte des ancêtres à la rentabilité des seniors : pour une anthropologique réaliste des (r)apports du troisième âge ». Texte inédit disponible sur http ://www.dvlp.ucl.ac.be/pic_ucl_uam/ms_1_culte_ ancetres_anthropologie_M_Singleton.html

TURNER, V. 1990. Le phénomène rituel. Structure et contre-structure, Paris, PUF (Édition originale, Aldindine Publishing, Company, 1969).

VAN GenNeP, A. 1909. Les rites de passage, Paris, Picard.

WitTGENSTEIN, L. 2006. De la certitude (notes posthumes écrites en 1951), Paris, Gallimard, coll. «Tel ».

WULF, C. (sous la direction de). 2004. Penser les pratiques sociales comme rituels. Ethnographie et genèse des communautés, Paris, L'Harmattan, coll. «Savoirs et formation». 